\title{
The Sun as a magnetic star: on the manifestation of different surface structures in disk-integrated observations
}

\author{
Mikhail L. Demidov \\ Institute of Solar-Terrestrial Physics, P.O. Box 291, 664033 Irkutsk, Russia \\ email: demid@iszf.irk.ru
}

\begin{abstract}
A reality of solar global magnetic fields Stokes-meter observations with polarimetric accuracy as high as $10^{-5}-10^{-6}$, and the dependence of Stokes $V$ profiles on distribution of surface magnetic fields in the central zone of the disk are demonstrated. A possibility to use solar disk-integrated and spatially resolved magnetic fields measurements for testing of magnetic fields mapping methods on Sun-like stars is suggested.
\end{abstract}

Keywords. Sun: magnetic fields - Sun: activity - polarization

The strength of solar magnetic fields strongly depends on the spatial resolution and place of observations. In the sunspot umbra we see thousands of Gauss, in the plages hundreds, in the quite atmosphere - only a few Gauss usually. But if we'll have a look on the Sun in the integrated radiation (in parallel beam of light or using defocusing of the image), we'll measure almost nothing. This "almost nothing" is the Solar Mean Magnetic Field (SMMF) or the magnetic field of the Sun as-a-star. Tremendous weakening of the measured strength originates from the low magnetic filling factor and non-coherency of solar magnetic fields, when opposite polarities cancel each other.

Cancel, but not perfectly, and if the accuracy of measurements is good enough, we can detect reliable Zeeman signal and explore the properties of the Sun as a magnetic star. Big scientific significance of such data is the reason why several observatories provide the regular or episodic measurements of disk-integrated solar magnetic fields. Up to now the only instrument which measures the SMMF in Stokes-meter regime (detection of the Stokes $I$ and $V$ profiles in several spectral lines simultaneously) is the STOP telescope at the Sayan Solar Observatory (Demidov et al., 2002). If the integration time is big enough, then a very weak signals could be detected (figure 1). Comparison of observations in different spectral lines shows that the SMMF strengths can differ by factor two or even more. Due to the limb darkening and some other reasons the main "contributor" to the SMMF signal is the central part of solar disk with radius $\approx 0.5$ of the full radius, and effect from limb zones, including polar caps, is negligible.

As other global solar parameters, the SMMF could (and must!) be used for testing of stellar observations. Indeed, recently a significant progress in measurements of magnetic fields on the other stars, including solar-type ones, is achieved (Petit et al., 2008). There are some promising attempts to map the surface distribution of stellar magnetic fields, using rotational modulation of the Stokes $V$ profiles. Simultaneous observations of the SMMF and full-disk solar magnetograms provide a unique chance for testing of such mapping algorithms. Figure 2 illustrates a strong dependence of the SMMF Stokes $V$ spectral lines profiles on the distribution across the solar disk (especially in the central zone) of the large-scale magnetic fields. 

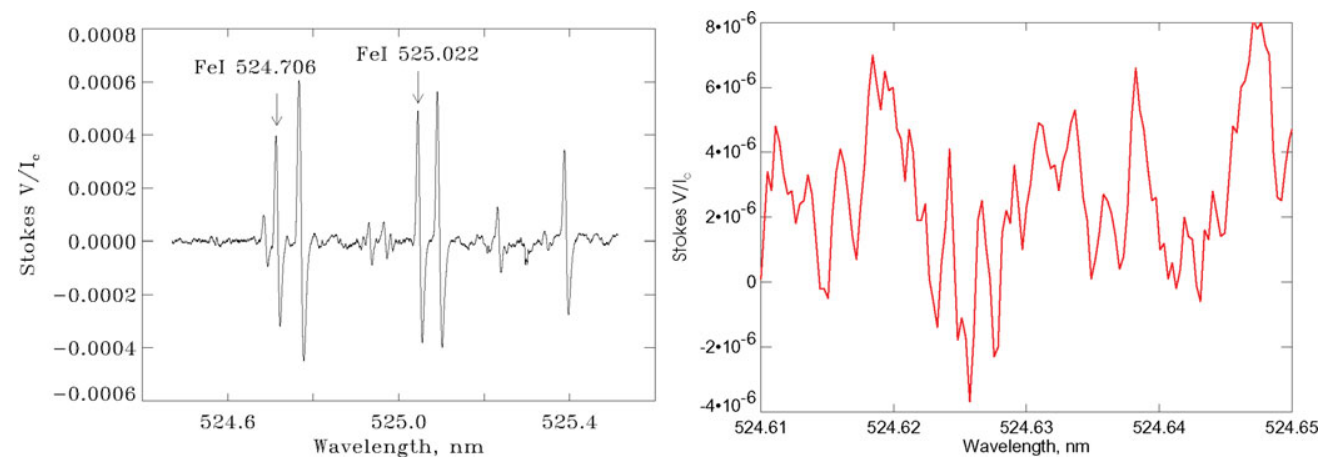

Figure 1. Left panel: the distribution of the Stokes $V / I_{c}$ parameter in the vicinity of spectral line Fe I $\lambda 525.02 \mathrm{~nm}$ in spectral band with width $\approx 1 \mathrm{~nm}$. Right panel: the distribution of the same parameter in the continuum. It is possibly to see, that the noise level is very small and such high degree of circular polarization as some units of $10^{-6}$ could be measured. Duration of SMMF observations is about 5 hours.
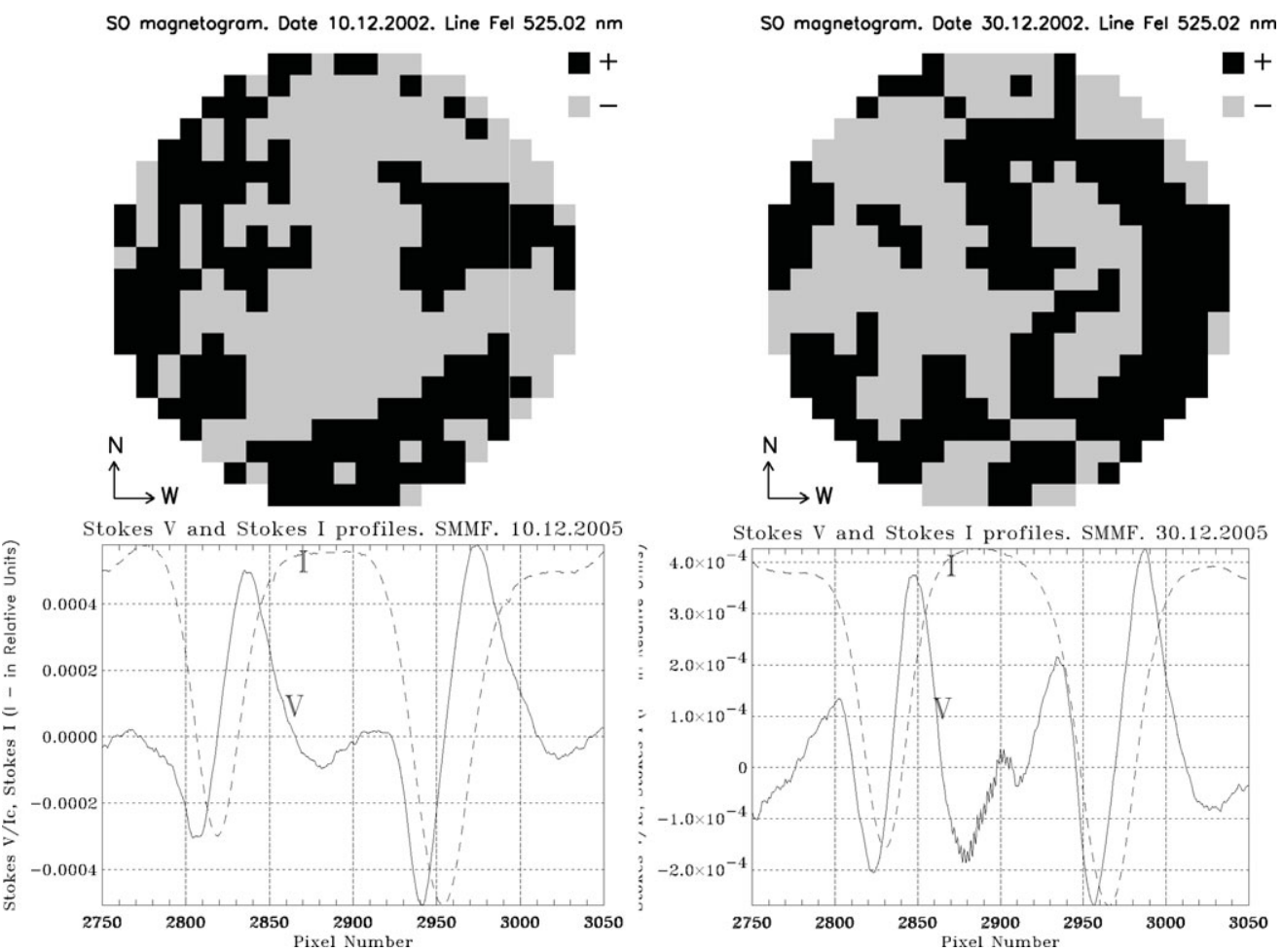

Figure 2. Illustration of the SMMF Stokes $V$ profiles (two bottom pictures) dependence on the structure of surface large-scale magnetic fields (two top pictures).

\section{Acknowledgements}

The author is grateful to the IAU S259 SOC and to the RFBR for financial support.

\section{References}

Demidov, M. L., Zhigalov, V. V., Peshcherov, V. S., \& Grigoryev, V. M. 2002, SP 209, 217

Petit, P., Ditrans, B., Solanki, S. K., et al. 2008, MNRAS 388, 80 